\title{
Preliminary Asteroseismology of EC20058-5234 and Limits on Plasmon Neutrinos
}

\author{
A. Bischoff-Kim \\ The University of Texas at Austin, Astronomy Department, 1 University Station, \\ C1400, Austin, TX 78712, USA
}

\begin{abstract}
We present results from a preliminary asteroseismological analysis of the hot DBV EC20058-5234 based on an extensive grid of models. EC20058 is the only known stable DBV and also the hottest and provides an ideal laboratory to constrain the emission rate of plasmon neutrinos. In a Whole Earth Telescope (WET) campaign in 1997, Sullivan et al. (2008) have found 11 independent modes. We use the results of their work to perform a 6 parameter fit of the observed period spectrum. We find a best fit model consistent with the spectroscopically determined effective temperature and surface gravity for EC20058. We find that the periods can be fit successfully without invoking an uneven split of the 281s mode due to a magnetic field. Based on our best fit model, we compute rates of change for the two stable modes observed in the star, which in turn can be used to place tight constraints on plasmon neutrino emission.
\end{abstract}

\section{Astrophysical Context}

Above a temperature of about $26000 \mathrm{~K}$, more than half of the luminosity of an average white dwarf comes from neutrino emission (Figure 1). In that temperature range, the neutrinos are mainly produced by the decay of photons coupled to a plasma (plasmons). If we measure a neutrino luminosity in hot white dwarfs, we are measuring plasmon neutrino rates. One can use the change in the pulsation periods over time to measure the neutrino luminosity. As a white dwarf cools, the period of a given mode increases because the interior is becoming less and less compressible. The faster the cooling, the faster the period increases. Mestel theory (Mestel 1952) predicts $\dot{P}$ if the white dwarf is leaking energy exclusively through photons. A higher $\dot{P}$ than expected means 
that the star is cooling faster than expected, and indicates an extra source of energy loss. $\dot{P}$ provides therefore a measure of the neutrino luminosity.

Following the discovery of pulsating DBs as hot as $28000 \mathrm{~K}$, Winget et al. (2004) demonstrated that one could use hot DBVs to measure neutrino rates. EC20058-05234 is the only stable DBV we know and for now we must rely on measuring $\dot{\mathrm{P}}$ for that star. EC20058 is also the hottest known DBV and as such, is a very good candidate for studying plasmon neutrino emission. Sullivan (2004) has collected over 10 years of data on EC20058 that can be used to determine the cooling rate of the star, though he has not published a $\dot{\mathrm{P}}$ yet.

We present preliminary results from an asteroseismological study of EC20058 and make a prediction of how tightly we can constrain the plasmon neutrino emission rates given a measured $\dot{\mathrm{P}}$ for EC20058. We begin with a brief introduction to plasmon neutrino physics before introducing EC20058's observed spectrum and what it tells us about the star's properties and the contribution of plasmon neutrino emission to its cooling.

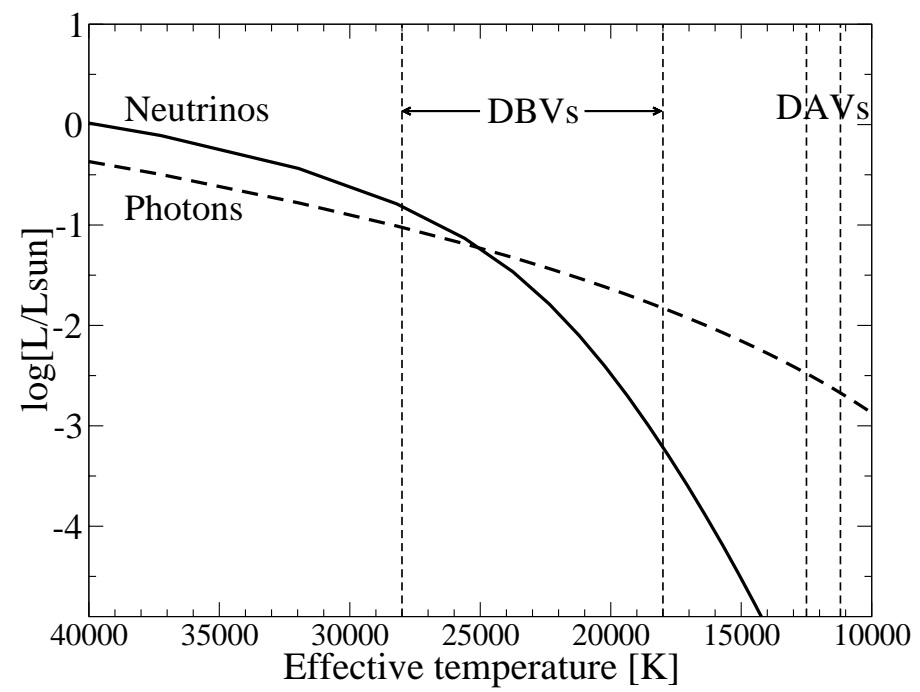

Figure 1: Time evolution of different sources of energy loss in a typical white dwarf model. The vertical dashed lines mark the location of the DBV and DAV instability strips. The neutrino luminosity remains significant near the hot (blue) edge of the DBV instability strip. 


\section{Plasmon neutrinos}

Plasmon neutrinos result from the decay of a plasmon into a neutrino-antineutrino pair. Classically, one thinks of a plasmon as an electromagnetic wave propagating through a dielectric medium. It is made up of an oscillating electromagnetic field coupled with electrons oscillating with the same frequency. The frequency, $\omega$, of such a wave obeys the dispersion relation

$$
\omega^{2}=\omega_{0}^{2}+k^{2} c^{2}
$$

where $\omega_{0}$ is the plasma frequency. Quantizing the field, the equation above yields

$$
E^{2}=\hbar^{2} \omega_{0}^{2}+p^{2} c^{2} .
$$

In effect, a plasmon is a particle similar to a photon, except it has a non-zero rest mass.

In free space, photons cannot decay into a neutrino-antineutrino pair without violating conservation of four-momentum. In a plasma, the electrons coupled to the photon allow conservation of energy and momentum and so plasmons can decay into a neutrino-antineutrino pair.

One can readily see from equation 1 that plasmons can only exist if $\omega>\omega_{0}$. This means that more plasmon neutrinos will be produced if the plasma has a high temperature relative to its frequency, i.e. $\mathrm{kT}>\hbar \omega_{0}$. However, $\omega$ should not be too large either because otherwise, the coupling with the electrons does not occur and plasmons do not exist. These conditions are satisfied in the interior of hot, neutrino emitting white dwarfs and is the dominant neutrino production process inside those stars.

\section{Observed properties of EC20058-5234}

EC20058 is the shortest period known DBV and the only one with stable pulsation periods. Spectroscopically, EC20058 appears to be hot and relatively low mass, with an effective temperature between $25600 \mathrm{~K}$ and $29900 \mathrm{~K}$ and a $\log (\mathrm{g})$ between 7.70 and 7.96 (Beauchamp et al. 1999). It is observable from the southern hemisphere and was first discovered to pulsate by Koen et al. (1995) from the South African Astronomical Observatory. In a 1997 Whole Earth Telescope (WET) run on this star, Sullivan et al. (2008) found 11 fundamental modes, listed in Table 1. The two highest amplitude modes $f 6$ (281.0s) and f8 (256.9s) are remarkably stable and may be used to measure a cooling rate for EC20058 and constrain plasmon neutrino emission rates.

Sullivan et al. (2008) found two modes each split by $70 \mu \mathrm{Hz}$. If we assume these modes are $\ell=1$ modes (a reasonable assumption from asymptotic period 
Table 1: Observed periods in EC20058

\begin{tabular}{|c|c|l|}
\hline $\begin{array}{c}\text { Mode name from } \\
\text { Sullivan et al. (2008) }\end{array}$ & Period $[\mathrm{s}]$ & Notes \\
\hline f1 & 539.8 & \\
f2 & 525.4 & \\
f3 & 350.6 & \\
f4 & 333.5 & \\
f5 & $(286.6)$ & m=-1 rotational split of f6 \\
f6 & 281.0 & High amplitude, stable mode \\
f7 & 274.7 & possible m=+1 split of f6 (see text) \\
f8 & 256.9 & High amplitude, stable mode \\
f16 & $(207.6)$ & m=-1 rotational split of f9 \\
f9 & 204.6 & \\
f11 & 195.0 & \\
\hline
\end{tabular}

spacing arguments and the relatively low mass found from spectroscopy), then the $70 \mu \mathrm{Hz}$ splitting is consistent with a rotation period of the star of 2 hours. Sullivan et al. (2008) also suggest that perhaps $f 7$ is the third member of the $(f 5, f 6, f 7)$ rotationally split $\ell=1$ triplet, invoking the existence of a $3 \mathrm{kG}$ magnetic field to account for the uneven frequency splitting. $f 7$ could also be a mode of its own that happens to lie close to where the $m=+1$ member of the $(f 5, f 6)$ multiplet would be if it were present. In the preliminary 6 parameter asteroseismological fits presented below, we consider both possibilities.

\section{Asteroseismological Fits}

In essence, the problem we have to solve in white dwarf asteroseismology is the simple minimization of a function (the average difference between the calculated periods and the observed periods) with $\mathrm{n}$ variables, including the stellar mass $\left(M_{*}\right)$, the effective temperature $\left(T_{\text {eff }}\right)$, and chosen structure parameters. Expressed mathematically:

$$
\Phi\left(\mathrm{T}_{\text {eff }}, \mathrm{M}_{*}, \ldots\right)=\sigma_{\mathrm{rms}}=\frac{1}{\mathrm{~N}} \Sigma_{\mathrm{i}=1}^{\mathrm{N}} \sqrt{\left(\mathrm{P}_{\mathrm{i}}^{\text {calc }}\right)^{2}-\left(\mathrm{P}_{\mathrm{i}}^{\mathrm{obs}}\right)^{2}},
$$

where $\mathrm{N}$ is the number of observed periods.

The simplest way to minimize $\Phi$ is to compute it for all conceivable values of the $n$ variables and pick the smallest value we find. But the number of times we 
have to evaluate $\Phi$ can quickly become astronomical. To make matters worse, each evaluation of the function requires the full computation of a white dwarf model. Fortunately, the White Dwarf Evolution Code (WDEC,Lamb \& Van Horn 1975, Wood 1990, see also Bischoff-Kim et al. 2008a for latest updates) allows us to compute a large number of models in a small amount of time on a standard desktop machine today and is perfect for the task.

To fit EC20058, we computed a grid of 286,416 models with simple core composition profiles and double-layered helium envelopes predicted by the time dependent diffusion of elements (Dehner \& Kawaler 1995, Althaus et al. 2005). We show the core composition profiles of a representative model in Figure 2. The associated structure parameters are the central oxygen abundance $\left(\mathrm{X}_{\mathrm{O}}\right)$, the location of the edge of the homegeneous carbon and oxygen core $\left(q_{\mathrm{fm}}\right)$, the location of the base of the helium rich layer $\left(\mathrm{M}_{\mathrm{env}}\right)$, and the location of the base of the pure helium layer $\left(\mathrm{M}_{\mathrm{He}}\right)$. Guided by the spectroscopy, we varied 6 parameters in the following ranges:

$\begin{array}{ccccc}25,600 \mathrm{~K} & \leq & T_{\text {eff }} & \leq & \leq 0,000 \mathrm{~K} \\ 0.45 \mathrm{M}_{\odot} & \leq & \mathrm{M}_{*} & \leq & 0.57 \mathrm{M}_{\odot} \\ -2 & \leq & \log \left(\mathrm{M}_{\text {env }}\right) & \leq & -4 \\ \log \left(\mathrm{M}_{\text {env }}\right)-2 & \leq & \log \left(\mathrm{M}_{\mathrm{He}}\right) & \leq & -8 \\ 0.5 & \leq & \mathrm{X}_{\mathrm{O}} & \leq & 1.0 \\ 0.35 \mathrm{M}_{*} & \leq & \mathrm{q}_{\mathrm{fm}} & \leq & 0.85 \mathrm{M}_{*}\end{array}$

We tried fits including 8 periods, making the assumption that $f 7$ in Table 1 is a member of the uneven triplet f5-f6-f7 due to a 2 hour rotation period of the star and a $3 \mathrm{kG}$ magnetic field; and a 9 periods fit, not invoking a magnetic field and including $f 7$ as a separate $m=0$ mode. We list the best fit found in the grid in both cases in Table 2 .

Table 2: Best fit parameters for EC20058

\begin{tabular}{|c|c|c|}
\hline Parameter & 8 period fit & 9 period fit \\
\hline$T_{\text {eff }}$ & $28,000 \mathrm{~K}$ & $28,400 \mathrm{~K}$ \\
$\mathrm{M}_{*}$ & $0.54 \mathrm{M}_{\odot}$ & $0.54 \mathrm{M}_{\odot}$ \\
$\log \left(\mathrm{M}_{\mathrm{env}}\right)$ & -3.6 & -3.6 \\
$\log \left(\mathrm{M}_{\mathrm{He}}\right)$ & -6.4 & -6.4 \\
$\mathrm{X}_{\mathrm{O}}$ & 1.00 & 0.80 \\
$\mathrm{q}_{\mathrm{fm}}$ & $0.35 \mathrm{M}_{*}$ & $0.35 \mathrm{M}_{*}$ \\
$\Phi$ & $1.92 \mathrm{~s}$ & $2.20 \mathrm{~s}$ \\
\hline
\end{tabular}



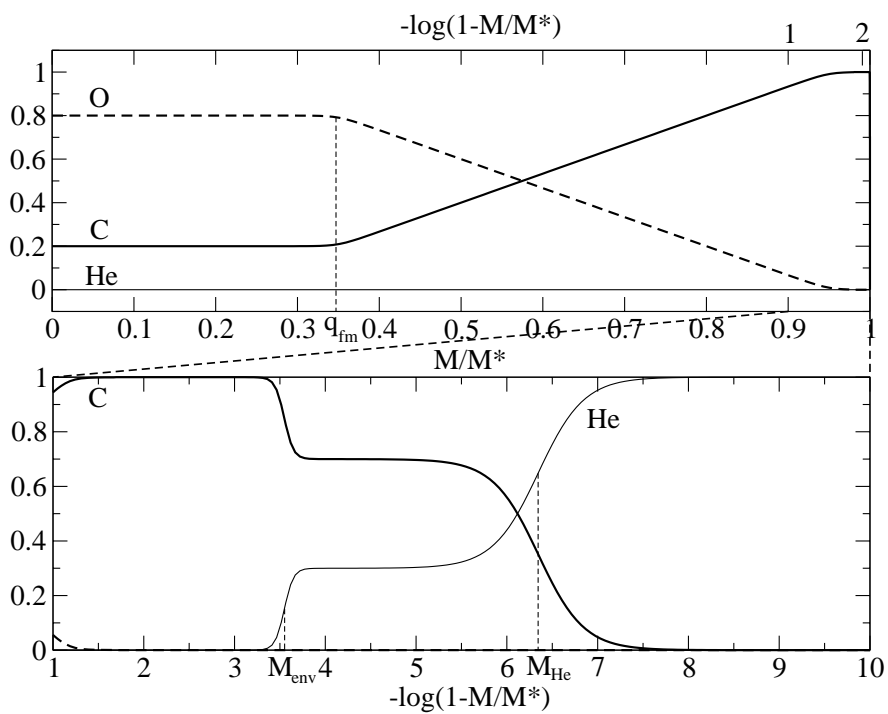

Figure 2: Core composition profiles for the grid models and internal structure parameters $\mathrm{q}_{\mathrm{fm}}, \mathrm{M}_{\mathrm{env}}, \mathrm{M}_{\mathrm{He}}$.

Both fits give similar answers and are equally good. While $\Phi$ for the 9 period fit is slightly higher, we have to compensate for the fact that we have added an extra period. Reduced $\chi^{2}$ 's and the Bayes information criterion (BIC, Koen \& Laney 2000) both indicate that the two fits are equivalent in quality. Occam's razor favors the 9 period fit (no need for a magnetic field in that case).

\section{Potential Limits on Plasmon Neutrino Rates}

We can now use the best fit model we found (for the 9 period fit) to calculate the rate at which the two stable modes (256.9 and 281.0s) change with time. We find $\dot{\mathrm{P}}_{256.9}=0.96 \times 10^{-13} \mathrm{~s} / \mathrm{s}$ and $\dot{\mathrm{P}}_{281.0}=1.4 \times 10^{-13} \mathrm{~s} / \mathrm{s}$. In order to study the effect of varying the plasmon neutrino rates, we define the parameter $\lambda$ as:

$$
\epsilon_{\nu}^{\prime}=\lambda \epsilon_{\nu}
$$

where $\epsilon_{\nu}$ is the plasmon neutrino emission rates given by ltoh et al. (1996) and $\epsilon_{\nu}^{\prime}$ is the changed rate used in the models.

We show P's as a function of varying plasmon neutrino rates for both stable modes in Figure 3. In the upper right corner of the left panel, we show the 
error bars we expect on the measuremed $\dot{\mathrm{P}}$ 's for these two modes. We estimate the error bars based on the ones we had in 1991 for G117-B15A $\left(0.35 \times 10^{-14}\right.$ s/s, Kepler et al. 1991), after 15 years of data had been collected on that star.

257s mode

281s mode

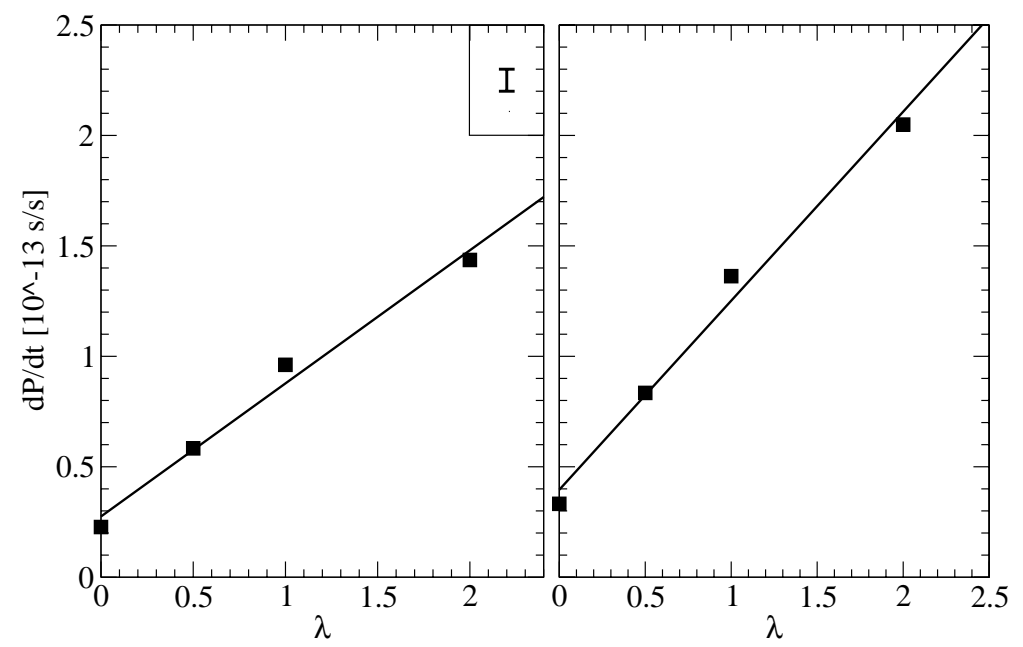

Figure 3: $\dot{\mathrm{P}}$ 's as a function of $\lambda$ (see equation 6 and text) for the two stable modes observed in EC20058; computed using the 9 period best fit model found in the previous section. In the box in the upper right corner of the left panel, we show the expected error bars on the measured P's.

\section{Conclusions}

Preliminary asteroseismological fits based on an extensive grid of models give best fit parameters consistent with the spectroscopic temperature and gravity determination for EC20058 and indicate that the star is likely oxygen rich. We obtain good fits without invoking a magnetic field to explain the $274.7 \mathrm{~s}$ mode observed in EC20058. From our best fit models we computed P's for the plasmon neutrino rates predicted by ltoh et al. (1996), as well as for modified plasmon neutrino rates. We have set the stage to constrain plasmon neutrino rates from EC20058's $\dot{\mathrm{P}}$. Once we have a measured $\dot{\mathrm{P}}$ for one or both of EC20058's stable modes, we should be able to place tight constraints on plas- 
mon neutrino emission rates. Most of the uncertainties lie in the models. More work is under way to improve the models and assess the associated uncertainties (Bischoff-Kim et al. 2008b).

Acknowledgments. This work was made faster and easier thanks to neatly packaged code provided by Dr. T. Metcalfe. This research was supported by NSF grant AST-0507639.

\section{References}

Althaus, L. G., Serenelli, A. M., Panei, J. A., et al. 2005, A\&A, 435, 631

Beauchamp, A., Wesemael, F., Bergeron, P., et al. 1999, ApJ, 516, 887

Bischoff-Kim, A., Montgomery, M. H., \& Winget, D. E. 2008a, ApJ, in press

Bischoff-Kim, A., Metcalfe, T. S., \& Montgomery, M. H. 2008b, in preparation

Dehner, B. T., \& Kawaler, S. D. 1995, ApJL, 445, L141

Itoh, N., Hayashi, H., Nishikawa, A., \& Kohyama, Y. 1996, ApJS, 102, 411

Kepler, S. O., Winget, D. E., Nather, R. E., et al. 1991, ApJL, 378, L45

Koen, C., O'Donoghue, D., Stobie, R. S., et al. 1995, MNRAS, 277, 913

Koen, C., \& Laney, D. 2000, MNRAS, 311, 636

Lamb, D. Q., \& van Horn, H. M. 1975, ApJ, 200, 306

Mestel, L. 1952, MNRAS, 112, 583

Sullivan, D. J. 2004, ASP Conf. Ser. 310: IAU Colloq. 193: Variable Stars in the Local Group, 310, 212

Sullivan, D. J., Metcalfe, T. S., O'Donoghue, D., et al. 2008, ApJ, submitted

Winget, D. E., Sullivan, D. J., Metcalfe, T. S., et al. 2004, ApJL, 602, L109

Wood, M. A. 1990, Ph.D. Thesis 


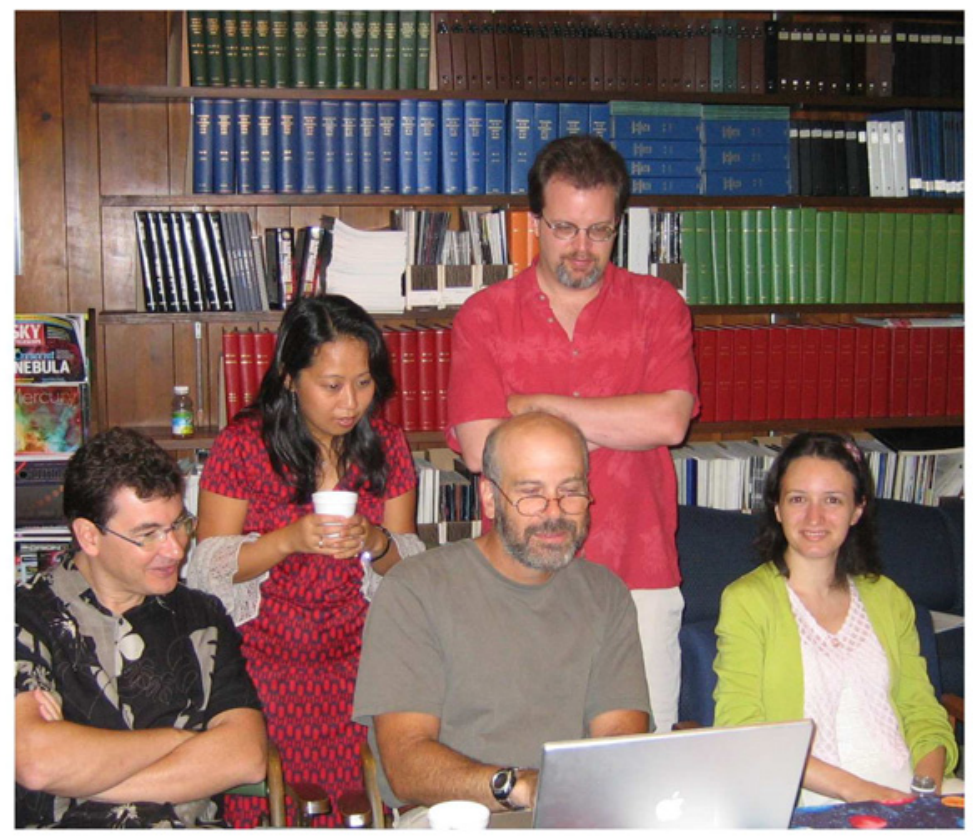

S. Kleinman, A. Nitta, R. Riddle and A. Kim-Bischoff gather around S. Kawaler's computer. 